\title{
Electrostatic Contributions to Chain Stiffness and Excluded-Volume Effects in Sodium Hyaluronate Solutions
}

\author{
Kanako Hayashi, Kikuko Tsutsumi, Takashi Norisuye, and Akio Teramoto \\ Department of Macromolecular Science, Osaka University, \\ Toyonaka, Osaka 560, Japan
}

(Received May 7, 1996)

\begin{abstract}
Intrinsic viscosities [ $\eta]$ for sodium hyaluronate in aqueous sodium chloride at $25^{\circ} \mathrm{C}$ were determined for 12 samples ranging in weight-average molecular weight $M_{w}$ from $3.8 \times 10^{3}$ to $3.5 \times 10^{5}$ at $\mathrm{NaCl}$ concentrations $C_{\mathrm{s}}$ between 0.005 and $2.5 \mathrm{M}$. They were analyzed on the basis of the Yamakawa-Fujii-Yoshizaki theory for [ $\eta$ ] of an unperturbed wormlike chain combined with the Yamakawa-Stockmayer-Shimada theory for excluded-volume effects to estimate the total persistence length $q$ and the excluded-volume strength $B$ as functions of $C_{\mathrm{s}}$. At any $C_{\mathrm{s}}$ studied, excluded-volume effects on [ $\left.\eta\right]$ became appreciable when $M_{w}$ exceeded $1 \times 10^{4}-2 \times 10^{4}$. The $C_{\mathrm{s}}$ dependence of $q$ yielded $4.0 \mathrm{~nm}$ for $q_{0}$ (the intrinsic persistence length) of the polysaccharide chain at infinite ionic strength. It was found that the values of $q-q_{0}$ (i.e., the electrostatic contribution to $q$ ) at $C_{\mathrm{s}}<0.02 \mathrm{M}$ were roughly $70 \%$ larger than predicted by the Le Bret theory and the Odijk-Skolnick-Fixman theory. On the other hand, the estimated $B$ values agreed fairly well with Fixman and Skolnick's theory for the excluded-volume interaction between a pair of charged rodlike segments unless $C_{\mathrm{s}}$ was lower than $0.05 \mathrm{M}$.
\end{abstract}

KEY WORDS Polyelectrolyte / Hyaluronic Acid / Wormlike Chain / Chain Stiffness / Electrostatic Persistence Length / Excluded-Volume Effect /

The current polyelectrolyte theory ${ }^{1-4}$ predicts that the persistence length $q$ of a charged linear polymer in aqueous salt increases with lowering salt concentration $C_{\mathrm{s}}$, provided the polyelectrolyte is modeled by the Kratky-Porod wormlike chain. ${ }^{5}$ This prediction for the electrostatic stiffening effect seems to be substantiated experimentally for intrinsically stiff polymers undergoing no appreciable intramolecular excluded-volume effect. Notably for double-stranded DNA, ${ }^{6}$ almost quantitative agreement was obtained between theoretical and experimental values for the electrostatic persistence length $q_{\mathrm{el}}$ (the electrostatic contribution to $q$ ).

On the other hand, our understanding of $q_{\mathrm{el}}$ for polyelectrolytes with intrinsically weak stiffness is far from satisfactory. The primary difficulty in the experimental determination of $q_{\mathrm{el}}$ or $q$ for those polymers is that the effects of chain stiffness and volume exclusion on measured properties can hardly be separated without resort to a relevant excluded-volume theory. Some previous workers ${ }^{7-9}$ deliberately or undeliberately ignored the latter effect in their estimation of $q$ and discussed apparent values so estimated for $q$ or $q_{\mathrm{el}}$. Others ${ }^{10-12}$ took account of volume effects, but they invoked the Fixman-Skolnick theory ${ }^{13}$ for the electrostatic binary cluster integral and early theories ${ }^{14,15}$ for the radius expansion factor $\alpha_{\mathrm{s}}$ based on the random flight model. Here, $\alpha_{s}$ is defined as the ratio of the perturbed to unperturbed radius of gyration. It is probably fair to say that no established way of estimating the chain stiffness is as yet known for intrinsically flexible or weakly stiff polyelectrolytes unless the ionic strength is high enough. We note that although scattering functions in the form of the Kratky plot are often considered to allow direct estimation of $q$, the method cannot always be justified even without excluded volume. ${ }^{16,17}$

The present study is concerned with our attempt at estimating $q$ and the excluded-volume strength (or the binary cluster integral) for hyaluronic acid (sodium salt) in aqueous $\mathrm{NaCl}$ at $25^{\circ} \mathrm{C}$ from measurements of the intrinsic viscosity $[\eta]$ covering a $C_{\mathrm{s}}$ range from 0.005 to $2.5 \mathrm{M}$. This polyacid consists of $N$-acetyl- $\beta$-Dglucosamine and $\beta$-D-glucuronic acid alternately linked at the 1,3 and 1,4 positions, respectively. Previous characterization work ${ }^{18,19}$ shows that its $\mathrm{Na}$ salt in 0.2 or $0.5 \mathrm{M}$ aqueous $\mathrm{NaCl}$ behaves like a weakly stiff wormlike chain with $q \sim 4 \mathrm{~nm}$ and undergoes significant intramolecular excluded-volume effects for molecular weight above $10^{4}-5 \times 10^{4}$. We here analyze the molecular weight dependence of $[\eta]$ for sodium hyaluronate at fixed $C_{\mathrm{s}}$ by the Yamakawa-Fujii-Yoshizaki theory $^{20.21}$ for the intrinsic viscosity $[\eta]_{0}$ of an unperturbed wormlike chain combined with the YamakawaStockmayer-Shimada (YSS) quasi-two-parameter theo$\mathrm{ry}^{22-24}$ for excluded-volume effects, assuming that without volume effect, the macroion at any $C_{\mathrm{s}}$ studied mimics the unperturbed wormlike chain regardless of its chain length. The results for $q$ and the excluded-volume strength thus obtained are compared with predictions from available polyelectrolyte theories. ${ }^{1-4,13}$ In regard to the adoption of the YSS theory, the following remarks are in order.

According to recent experimental studies, ${ }^{25,26}$ data of $\alpha_{s}{ }^{2}$ and $\alpha_{\eta}{ }^{3}$ (the cube of the viscosity expansion factor defined by $\left.[\eta] /[\eta]_{0}\right)$ for uncharged polymers, either flexible or stiff, are explained almost quantitatively in the framework of the YSS theory, i.e., by the YSS perturbation theory for the wormlike or helical wormlike chain $^{27}$ combined with the Domb-Barrett function ${ }^{28}$ for $\alpha_{s}{ }^{2}$ or the Barrett function ${ }^{29}$ for $\alpha_{\eta}^{3}$. This quasi-twoparameter theory is also a good approximation to $\mathrm{Na}$ hyaluronate in 0.2 and $0.5 \mathrm{M}$ aqueous $\mathrm{NaCl}^{19,26}$ Thus, it seems to be the most relevant to our purpose at present, though its applicability to polyelectrolytes at low ionic strength remains to be seen. 


\section{EXPERIMENTAL}

\section{Samples}

The previously investigated 12 fractions ${ }^{19}$ of $\mathrm{Na}$ hyaluronate, designated HA1, HA2, $\cdots$, and HA12 in order of decreasing molecular weight, were used for the present study. Their weight-average molecular weights $M_{w}$ (determined by light scattering or sedimentation equilibrium in $0.5 \mathrm{M}$ aqueous $\mathrm{NaCl}$ ) ranged from $3.8 \times 10^{3}$ to $3.5 \times 10^{5}$ (see Table I). The ratios of the $z$-average to weight-average molecular weight for six samples HA6 through HA11 were about 1.1. ${ }^{19}$

\section{Viscometry}

Aqueous $\mathrm{NaCl}$ solutions of $\mathrm{Na}$ hyaluronate were prepared as described previously, ${ }^{19}$ and their zero-shear rate viscosities at $25^{\circ} \mathrm{C}$ were determined at $C_{\mathrm{s}}=0.005$, $0.0067,0.01,0.02,0.05,0.1,1$, and $2.5 \mathrm{M}$; for the lowest molecular weight sample HA12, the measurement was made only at $C_{\mathrm{s}}=0.005$ and $0.01 \mathrm{M}$ because of the limited sample quantity. A four-bulb low-shear capillary viscometer of the Ubbelohde type was used for sample HA1 at every $C_{\mathrm{s}}$ and for sample HA2 at $C_{\mathrm{s}} \leq 0.01 \mathrm{M}$, and conventional capillary viscometers for the rest; we note that shear-rate effects on $[\eta]$ were small (less than $1.5 \%$ ) for $[\eta]<11 \times 10^{2} \mathrm{~cm}^{3} \mathrm{~g}^{-1}$ when $[\eta]$ values from the two types of viscometer were compared. As in our previous work, the relative viscosity was evaluated by taking account of the difference between the solution and solvent densities for $[\eta]<60 \mathrm{~cm}^{3} \mathrm{~g}^{-1}$. The Huggins plot, the Fuoss-Mead plot, and the Billmeyer plot were combined to determine $[\eta]$ and Huggins' constant $k^{\prime}$. For the three lowest molecular weight samples at some low salt concentrations, however, precise values of $k^{\prime}$ could not be determined because the measurements were confined to low relative viscosities.

The $[\eta]$ data obtained were considered free from electroviscous effects. The reason for this was as follows.

According to Sho et al., ${ }^{30}$ the double helix of $\mathrm{Na}$ xanthan (linear charge density $=3 \mathrm{~nm}^{-1}$ ) in aqueous $\mathrm{NaCl}$ at $25^{\circ} \mathrm{C}$ has an intrinsically high rigidity as characterized by a $q$ of about $110 \mathrm{~nm}$ at infinite ionic strength. This implies that the polymer is hardly stiffened by lowering $C_{\mathrm{s}}$. In other words, without electroviscous effect, $[\eta]$ for double-helical xanthan with a relatively low molar mass should be independent of $C_{\mathrm{s}}$. The $[\eta]$ data of Sho et al. indeed show this to be the case for $\mathrm{Na}$ xanthan samples with $M_{w}$ of $2 \times 10^{5}-1 \times 10^{6}$ over a $C_{\mathrm{s}}$ range from 0.005 to $1 \mathrm{M}$.

\section{RESULTS}

Numerical data of zero-shear-rate $[\eta]$ for $\mathrm{Na}$ hyaluronate in aqueous $\mathrm{NaCl}$ are summarized in Table $\mathrm{I}$, in which those at $C_{\mathrm{s}}=0.2$ and $0.5 \mathrm{M}$ are the reproductions from our previous paper. ${ }^{19}$ All these $[\eta]$ values are plotted double-logarithmically against $C_{\mathrm{s}}$ in Figure 1. The curve fitting the plotted points for each sample rises with decreasing $C_{\mathrm{s}}$, but it flattens as the molecular weight decreases.

Figure 2 shows the $C_{\mathrm{s}}$ dependence of $k^{\prime}$ for samples HA1 through HA9. The values of $k^{\prime}$ for the three highest molecular weight samples are essentially constant

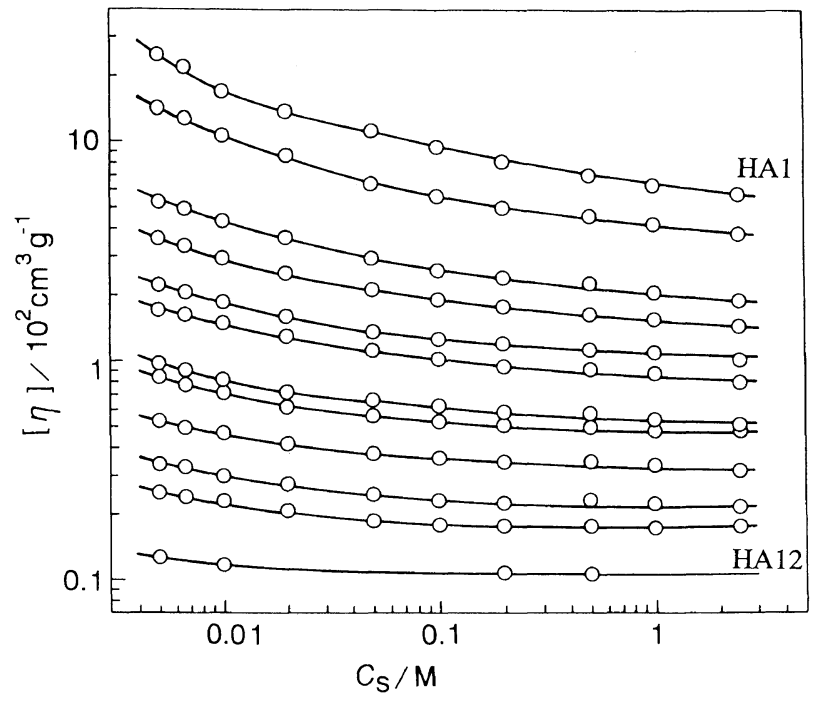

Figure 1. Dependence of $[\eta]$ on $C_{\mathrm{s}}$ for Na hyaluronate samples (HA1, HA2, $\cdots, \mathrm{HA} 12$ from top to bottom) in aqueous $\mathrm{NaCl}$ at $25^{\circ} \mathrm{C}$. The data at $C_{\mathrm{s}}=0.2$ and $0.5 \mathrm{M}$ are the reproductions from ref 19 .

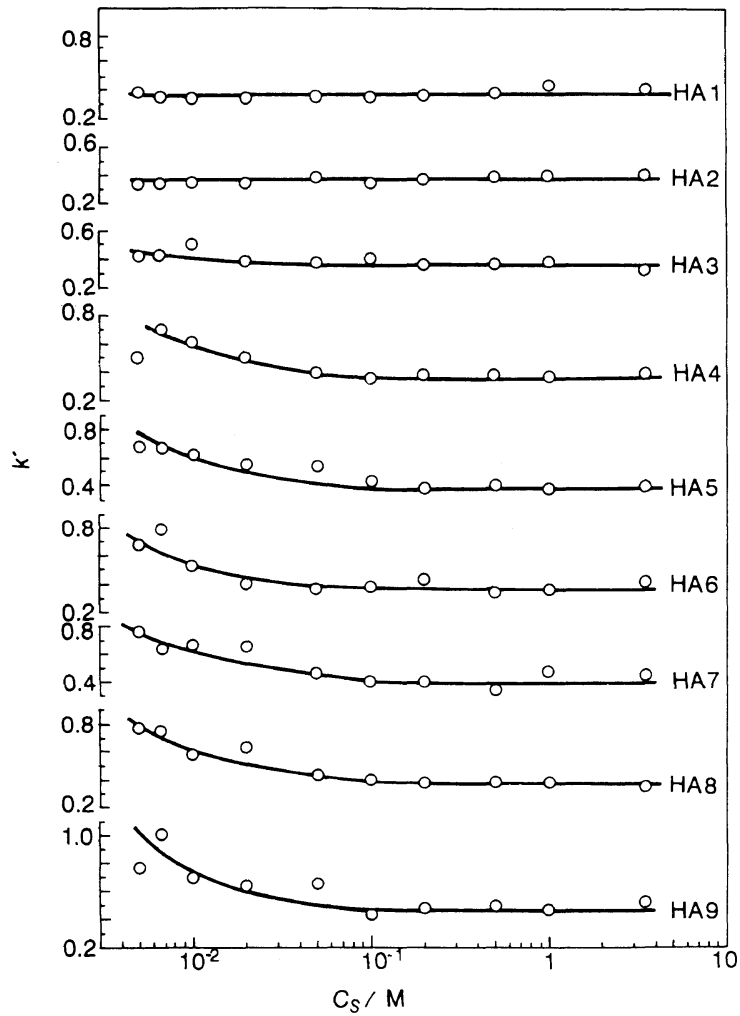

Figure 2. Huggins' constant plotted against $\log C_{\mathrm{s}}$ for indicated $\mathrm{Na}$ hyaluronate samples in aqueous $\mathrm{NaCl}$ at $25^{\circ} \mathrm{C}$. The data at $C_{\mathrm{s}}=0.2$ and $0.5 \mathrm{M}$ were obtained in our previous work. ${ }^{19}$

throughout the entire range of $C_{\mathrm{s}}$ studied. Those for the other samples stay almost constant down to $C_{\mathrm{s}} \sim 0.1 \mathrm{M}$ and then sharply increase with decreasing $C_{\mathrm{s}}$. Similar $M_{w}$-dependent increases in $k^{\prime}$ were observed by Sho et $a l .{ }^{30}$ for $\mathrm{Na}$ salt xanthan in aqueous $\mathrm{NaCl}$.

Figure 3 depicts the molecular weight dependence of $[\eta]$ at the indicated salt concentrations. The plotted points for each $C_{\mathrm{s}}$ follow a curve which is slightly convex upward for $M_{w}<10^{4}$ and roughly linear for $M_{w}>10^{4}$. The slope in this high $M_{w}$ region is 0.84 at the highest $C_{\mathrm{s}}$ of $2.5 \mathrm{M}$ and 1.14 at the lowest $C_{\mathrm{s}}$ of $0.005 \mathrm{M}$. Even 


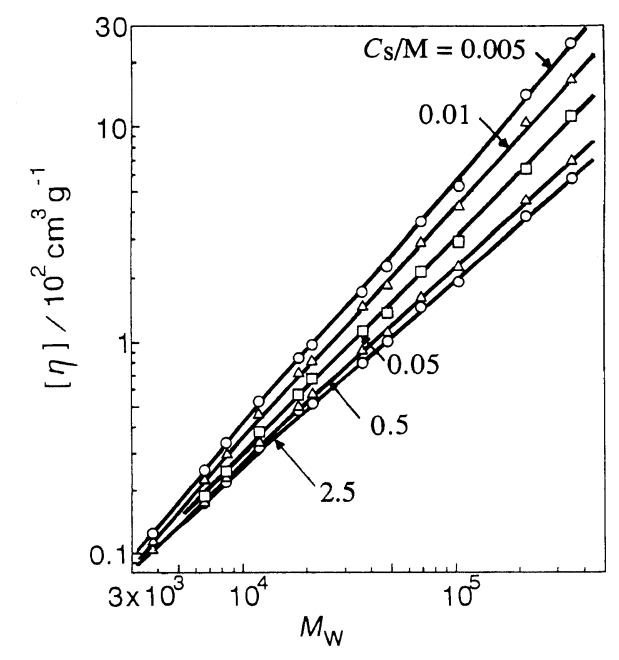

Figure 3. Molecular weight dependence of $[\eta]$ for $\mathrm{Na}$ hyaluronate in aqueous $\mathrm{NaCl}$ with indicated $C_{\mathrm{s}}$ at $25^{\circ} \mathrm{C}$.

the former viscosity exponent is slightly larger than the upper bound value of 0.80 predictable for uncharged flexible chains.

\section{DISCUSSION}

Data Analysis

Within the framework of the YSS theory ${ }^{2-24}$ for nonionic wormlike or helical wormlike bead chains, $\alpha_{\eta}{ }^{3}$ is a universal function of the scaled excluded-volume parameter $\tilde{z}$ defined by

$$
\tilde{z}=(3 / 4) K(\lambda L) z
$$

with

$$
z=(3 / 2 \pi)^{3 / 2}(\lambda B)(\lambda L)^{1 / 2}
$$

and

$$
\begin{aligned}
K(\lambda L)= & (4 / 3)-2.711(\lambda L)^{-1 / 2} \\
& +(7 / 6)(\lambda L)^{-1} \text { for } \lambda L>6 \\
= & (\lambda L)^{-1 / 2} \exp \left[-6.611(\lambda L)^{-1}\right. \\
& +0.9198+0.03516 \lambda L] \\
& \text { for } \lambda L \leq 6
\end{aligned}
$$

Here, $\lambda^{-1}$ is the stiffness parameter, $L$ is the contour length of the chain related to the molecular weight $M$ by $M_{\mathrm{L}}=M / L$, with $M_{\mathrm{L}}$ being the molar mass per unit contour length, $z$ is the conventional excluded-volume parameter, and $B$ is the excluded-volume strength for the interaction between a pair of beads. The $K(\lambda L)$ function is essentially zero for $\lambda L$ below unity due to the stiffness effect and approaches the coil-limiting value of $4 / 3$ with increasing $\lambda L$. For the wormlike chain (a special limit of the helical wormlike chain ${ }^{27}$ ) we have

$$
\begin{aligned}
& \lambda^{-1}=2 q \\
& B=\beta / a^{2}
\end{aligned}
$$

where $\beta$ and $a$ are the binary cluster integral and the bead spacing, respectively. The Barrett function ${ }^{29}$ in the YSS scheme reads ${ }^{31}$

$$
\alpha_{\eta}{ }^{3}=\left(1+3.8 \tilde{z}+1.9 \tilde{z}^{2}\right)^{0.3}
$$

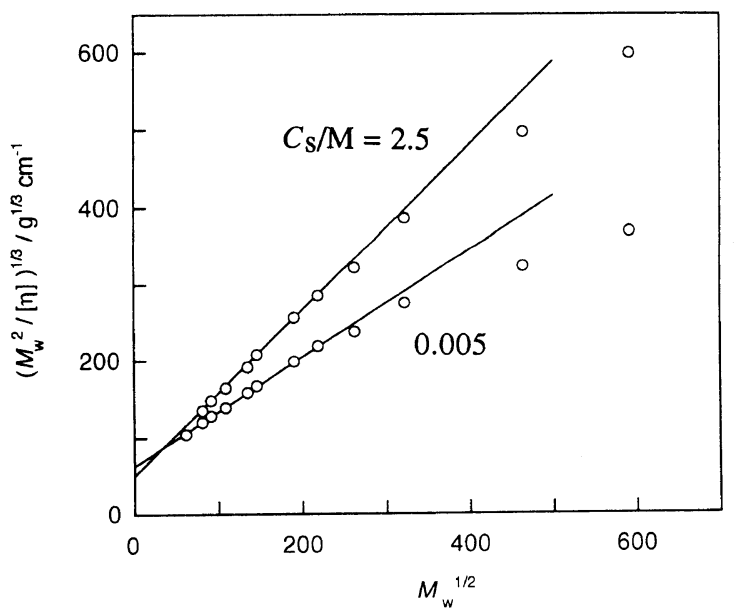

Figure 4. Bushin-Bohdanecký plots for $\mathrm{Na}$ hyaluronate in 0.005 and $2.5 \mathrm{M}$ aqueous $\mathrm{NaCl}$ at $25^{\circ} \mathrm{C}$.

which reduces to the original Barrett equation in the coil limit where $\tilde{z}=z$.

The Yamakawa-Fujii-Yoshizaki theory ${ }^{20,21}$ for $[\eta]_{0}$ of an unperturbed wormlike chain contains $M_{\mathrm{L}}, q$, and $d$ (the hydrodynamic diameter in the cylinder model) as the parameters, so that $[\eta]$ in a perturbed state is determined by $M_{\mathrm{L}}, q, d$, and $B$; note that this theory is essentially equivalent to that ${ }^{32}$ based on the touchedbead model if the difference in hydrodynamic diameter is taken into account. In the data analysis presented below, we first determine $M_{\mathrm{L}}$ of $\mathrm{Na}$ hyaluronate at each $C_{\mathrm{s}}$ from $[\eta]$ data at relatively low $M_{w}$ without explicit consideration of excluded-volume effects and then $q$ and $B$ from all $[\eta]$ data with their consideration, assuming that the $d$ value of $1.0 \mathrm{~nm}$ estimated previously ${ }^{19}$ at $C_{\mathrm{s}}=0.2 \mathrm{M}$ is applicable to any other $C_{\mathrm{s}}$.

Figure 4 illustrates the Bushin-Bohdanecký plots ${ }^{33,34}$ of $\left(M_{w}{ }^{2} /[\eta]\right)^{1 / 3}$ against $M_{w}{ }^{1 / 2}$ at $C_{\mathrm{s}}=0.005$ and $2.5 \mathrm{M}$, constructed according to the equation (an approximation to the Yamakawa-Fujii-Yoshizaki theory)

$$
\left(M^{2} /[\eta]_{0}\right)^{1 / 3}=I+S M^{1 / 2}
$$

where $I$ and $S$ are given by

$$
\begin{gathered}
I=1.516 \times 10^{-8} I_{0} M_{\mathrm{L}}\left(\mathrm{g}^{1 / 3} \mathrm{~cm}^{-1}\right) \\
S=1.516 \times 10^{-8} S_{0}\left(M_{\mathrm{L}} / 2 q\right)^{1 / 2}\left(\mathrm{~g}^{1 / 3} \mathrm{~cm}^{-1}\right)
\end{gathered}
$$

with $I_{0}$ and $S_{0}$ being known functions of $d / 2 q$. The plotted points for either $C_{\mathrm{s}}$ are seen to follow a straight line below $M_{w}{ }^{1 / 2} \sim 220$, but above that they deviate downward from the line progressively with increasing $M_{w}{ }^{1 / 2}$, showing pronounced intramolecular excluded-volume effects. Plots for other $C_{\mathrm{s}}$ exhibited similar trends. The intercept $I$ and slope $S$ for each $C_{\mathrm{s}}$ together with $d=$ $1.0 \mathrm{~nm}$ yield a set of $M_{\mathrm{L}}$ and $q$ values, but, as mentioned above, we re-evaluate $q$ in such a way that for $d=1.0 \mathrm{~nm}$ and the estimated $M_{\mathrm{L}}$ value, a set of $q$ and $B$ allows the Yamakawa-Fujii-Yoshizaki theory with eq 6 to give the closest fit to the $[\eta]$ data in the entire $M_{w}$ range studied; note that, as remarked previously, ${ }^{19}$ stiffness and excluded-volume effects may lead to similar chain length dependences of $[\eta]$ in a certain, narrow molecular weight range. 
The values of $M_{\mathrm{L}}, q$, and $B$ thus obtained are summarized in Table II, along with the previous results at $C_{\mathrm{s}}=0.2$ and $0.5 \mathrm{M}$, and in Figure 5 , the best fit theoretical curves (the solid lines) are compared with the experimental data at the indicated salt concentrations. These solid curves appear somewhat above the data points for $M_{w}$ between $3 \times 10^{4}$ and $1.5 \times 10^{5}$ and somewhat below those for $M_{w}>2 \times 10^{5}$, but except for $C_{\mathrm{s}}=0.005 \mathrm{M}$, the general agreement is fairly good. The dashed lines in the figure represent the theoretical $[\eta]_{0}$ values for $C_{\mathrm{s}}=0.005$ and $2.5 \mathrm{M}$. Excluded-volume effects on $[\eta]$ at either $C_{\mathrm{s}}$ are seen to become appreciable at a molecular weight of $1 \times 10^{4}-2 \times 10^{4}$. The same was true for all the other salt concentrations. With regard to the poor agreement between the theoretical and experimental $[\eta]$ values at $C_{\mathrm{s}}=0.005 \mathrm{M}$, a few remarks may be made.

1. As already mentioned, the experimental log $[\eta]$ vs. $\log M_{w}$ relation at $C_{\mathrm{s}}=0.005 \mathrm{M}$ is almost linear for $M_{w}>1 \times 10^{4}$ with a viscosity exponent of 1.14 . The contribution from $[\eta]_{0}$ to this exponent is about 0.79 in

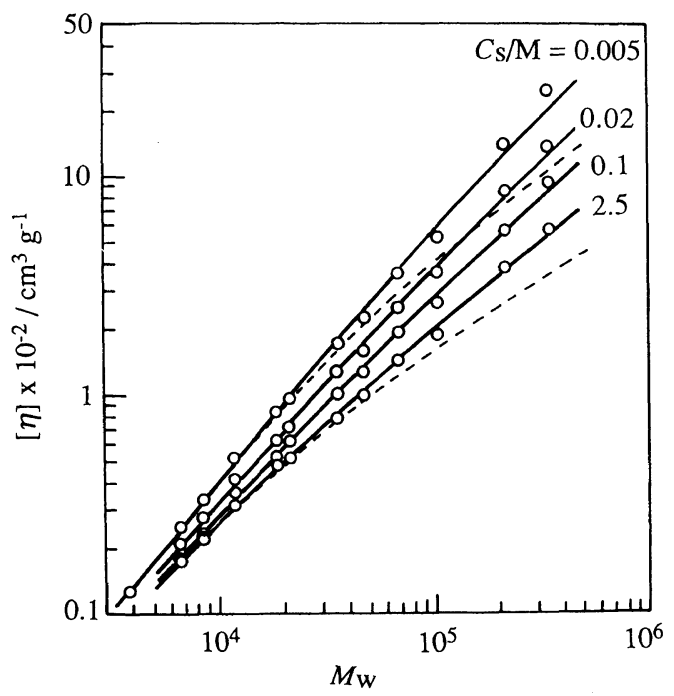

Figure 5. Comparison between the measured $[\eta]$ for $\mathrm{Na}$ hyaluronate at indicated $C_{\mathrm{s}}$ (the circles) and the theoretical values (the solid lines) calculated from the YSS theory (the Yamakawa-Fujii-Yoshizaki theory for $[\eta]_{0}+$ eq 6 ) for perturbed wormlike chains with $d=1.0 \mathrm{~nm}$ and the parameters in Table II. The two dashed lines refer to the unperturbed wormlike chains at $C_{\mathrm{s}}=0.005$ (upper) and $2.5 \mathrm{M}$ (lower). the molecular weight range between $6 \times 10^{4}$ and $4 \times 10^{5}$ when estimated from the dashed line (for $C_{\mathrm{s}}=0.005 \mathrm{M}$ ) in Figure 5. The difference of 0.35 in exponent, which comes from excluded-volume effects, exceeds the asymptotic value 0.30 predicted by eq 6 , though the theoretical exponent for $\alpha_{\eta}^{3}$ can be somewhat larger than 0.3 at finite $L$ because of the $L$ dependence of $K(\lambda L)$ in $\tilde{z}$ (see eq 3 ). A similar argument was found to apply to $C_{\mathrm{s}}=0.0067 \mathrm{M}$, at which the agreement between the theoretical and experimental $[\eta]$ for $M_{w}>4 \times 10^{4}$ was not good either. Thus the high experimental exponents for $\alpha_{\eta}{ }^{3}$ seem to be responsible for the poor agreements between the YSS theory and the present data at the two lowest salt concentrations, suggesting need of some specific theoretical consideration for electrostatic excluded-volume interactions at such low ionic strength.

2. Because of theses poor agreements, the values of $B$ at $C_{\mathrm{s}}=0.005$ and $0.0067 \mathrm{M}$ in Table II are inaccurate. They can be larger by $30 \%$ or more, provided that even at these low $\mathrm{NaCl}$ concentrations eq 6 is valid for very high molecular weights.

3. On the other hand, the $q$ values at the two lowest salt concentrations do not seem to rely strongly on the YSS theory since, as may be seen in Figure 5, both solid and dashed lines for $C_{\mathrm{s}}=0.005 \mathrm{M}$ fit the data points up to $M_{w} \sim 2 \times 10^{4}$ and their difference remains rather small up to $M_{w} \sim 5 \times 10^{4}$. In actuality, the estimated $q$ value of $9.7 \mathrm{~nm}$ at this $C_{\mathrm{s}}$ (see Table II) differs only by $8 \%$ from that $(10.5 \mathrm{~nm})$ pre-determined by use of the Bushin-Bohdanecký plot in Figure 4. Furthermore, if a smaller $q$ of $9.0 \mathrm{~nm}$ is chosen instead of $9.7 \mathrm{~nm}$, the fit of the YSS curve in the region of $M_{w}$ below $2 \times 10^{4}$ becomes definitely worse regardless of the value of $B$. Thus the errors of our $q$ values at $C_{\mathrm{s}}=0.0067$ and $0.005 \mathrm{M}$ should be less than $10 \%$.

In Table II, the $M_{\mathrm{L}}$ values are all in the range between 400 and $410 \mathrm{~nm}^{-1}$. They are consistent with the chemical structure of $\mathrm{Na}$ hyaluronate, ${ }^{18,19}$ indicating that the wormlike chain is a good model for $\mathrm{Na}$ hyaluronate in the $C_{\mathrm{s}}$ range studied. It can also be seen in the table that $q$ and $B$ monotonically increase with lowering $C_{\mathrm{s}}$. This shows that both stiffness and volume effects are responsible for the increases in $[\eta]$ with decreasing $C_{\mathrm{s}}$ observed for high molecular weight samples in Figure 1.

Table I. Intrinsic viscosities for sodium hyaluronate samples in aqueous $\mathrm{NaCl}$ with different salt concentrations at $25^{\circ} \mathrm{C}$

$[\eta] \times 10^{-2} / \mathrm{cm}^{3} \mathrm{~g}^{-1}$

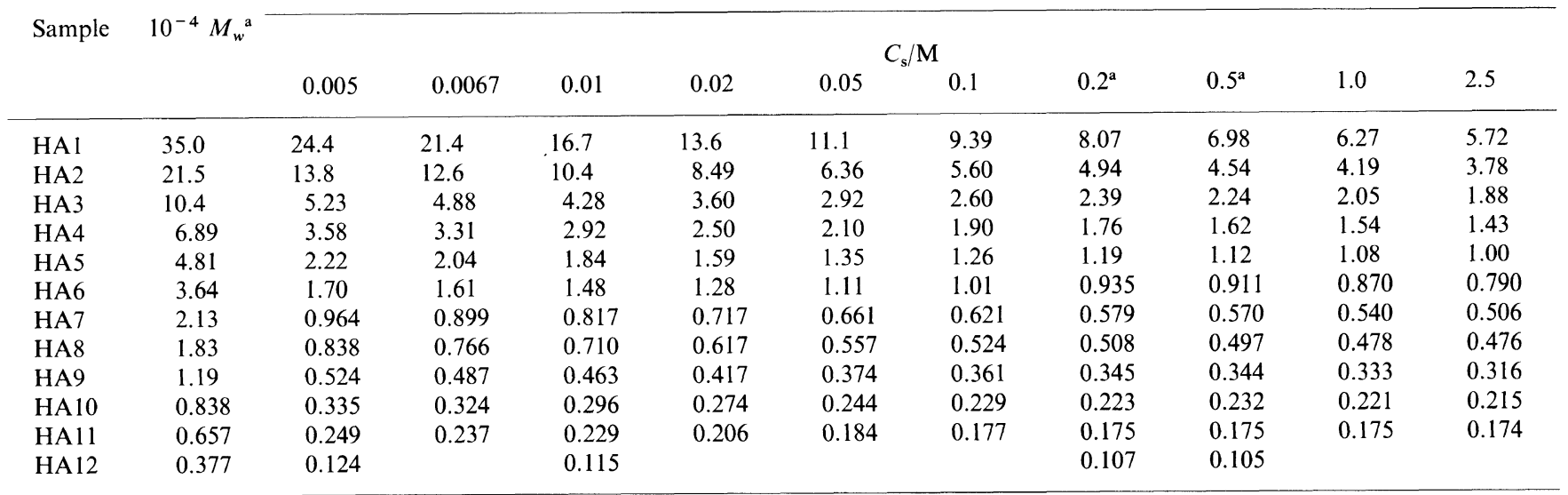

${ }^{a}$ Taken from ref 19. 
For $C_{\mathrm{s}}>0.2 \mathrm{M}$, however, $q$ hardly changes with $C_{\mathrm{s}}$ while $B$ increases with decreasing $C_{\mathrm{s}}$. Thus, in this high $C_{\mathrm{s}}$ region, the observed viscosity increases may be ascribed primarily to excluded-volume effects.

\section{Electrostatic Contribution to the Persistence Length}

Odijk $^{1}$ and Skolnick and Fixman ${ }^{2}$ independently showed that $q$ of a charged wormlike chain is the sum of $q_{0}$ (the intrinsic persistence length) and $q_{\mathrm{el}}$, i.e., $q=q_{0}+q_{\mathrm{el}}$. These authors derived an analytical expression for $q_{\mathrm{el}}$ of a thin wormlike chain with line charge distribution on the basis of the Debye-Hückel approximation to the Poisson-Boltzmann equation. More elaborate analyses were made by Le Bret $^{3}$ and Fixman, ${ }^{4}$ who computed $q_{\mathrm{el}}$ for a wormlike cylinder with radius $r$, dielectric constant $\varepsilon$, and smeared charge distribution (on the surface), using the complete Poisson-Boltzmann equation. The resulting $q_{\mathrm{el}}$ values are tabulated in Le Bret's paper as a function of $\sigma$ (the linear charge density) and $\kappa r$ for $\varepsilon / \varepsilon_{0}=0$ and $\infty$. Here, $\varepsilon_{0}$ is the dielectric constant of water and $\kappa$ is the reciprocal of the Debye screening length defined by $\kappa^{2}=8 \pi Q N_{\mathrm{A}} C_{\mathrm{s}} / 1000$ for aqueous $1-1$ electrolyte, with $Q$ and $N_{\mathrm{A}}$ being the Bjerrum length $\left(0.714 \mathrm{~nm}\right.$ for water at $\left.25^{\circ} \mathrm{C}\right)$ and the Avogadro constant, respectively; $C_{\mathrm{s}}$ is expressed in units of M. All these theories predict $q_{\mathrm{el}}$ to be a decreasing function of $C_{\mathrm{s}}$ and vanish at infinite ionic strength, so that $q$ at this limit equals $q_{0}$. From the data of $q$ in Table II we estimate $q_{0}$ of $\mathrm{Na}$ hyaluronate (at infinite ionic strength) to be $4.0 \mathrm{~nm}$.

In Figure 6, our $q$ data are compared with the Le Bret theory ${ }^{3}$ for $q_{0}=4.0 \mathrm{~nm}, \sigma=1.0 \mathrm{~nm}^{-1}, \varepsilon / \varepsilon_{0}=0$, and $r=0.5 \mathrm{~nm} ; q_{0}$ has been taken to be independent of $C_{\mathrm{s}}$ in first approximation and the $\sigma$ value has been evaluated from the average $M_{\mathrm{L}}$ of $405 \mathrm{~nm}^{-1}$ (see Table II) and the molar mass $\left(401 \mathrm{~g} \mathrm{~mol}^{-1}\right)$ of the repeating unit of $\mathrm{Na}$ hyaluronate. The theoretical $q_{\mathrm{el}}$ values are about $70 \%$ smaller than the experimental ones for $C_{\mathrm{s}}{ }^{-1 / 2}>7 \mathrm{M}^{-1 / 2}$ (i.e., for $C_{\mathrm{s}}<0.02 \mathrm{M}$ ), the discrepancy being much larger than possible errors in our estimation of $q$. Interestingly, however, both theoretical and experimental $q_{\mathrm{el}}$ 's, when plotted double-logarithmically against $C_{\mathrm{s}}$ (not shown), are essentially parallel with a slope of -0.6 for $C_{\mathrm{s}}<$ $0.1 \mathrm{M}$. This slope happens to be close to those (about -0.5 ) estimated for several intrinsically flexible or weak-

Table II. Wormlike chain parameters and excluded-volume strength for sodium hyaluronate in aqueous $\mathrm{NaCl}$ at $25^{\circ} \mathrm{C}$

\begin{tabular}{lcccc}
\hline \multicolumn{1}{c}{$C_{s}$} & \multicolumn{2}{c}{$M_{\mathrm{L}}$} & & \multicolumn{2}{c}{$B$} \\
\cline { 5 - 5 } & $\mathrm{nm}^{-1}$ & & $\mathrm{~nm}$ & $\mathrm{~nm}$ \\
\hline 0.005 & 400 & 9.7 & 16 \\
0.0067 & 400 & & 8.5 & 14 \\
0.01 & 400 & 7.4 & 11 \\
0.02 & 405 & 6.0 & 9.0 \\
0.05 & 410 & 5.0 & 6.0 \\
0.1 & 410 & 4.8 & 4.8 \\
$0.2^{\mathrm{a}}$ & 405 & 4.2 & 3.4 \\
$0.5^{\mathrm{a}}$ & 400 & 4.1 & 2.8 \\
1 & 400 & 4.0 & 2.3 \\
2.5 & 405 & 4.0 & 1.6
\end{tabular}

a Taken from ref 19 . ly stiff polyelectrolytes ${ }^{7,11}$ without consideration of excluded-volume effects.

The Odijk-Skolnick-Fixman (OSF) theory predicts the $C_{s}^{-1}$ dependence (or more generally the $\kappa^{-2}$ dependence) of $q_{\mathrm{el}}$ differing from the Le Bret theory, but it (without ion condensation ${ }^{35)}$ gives $q$ values close to the Le Bret line, as indicated by a dashed curve in Figure 6 ; note that the charge parameter $\sigma Q$ for the hyaluronate chain is less than unity (about 0.7). Skolnick and Fixman's calculation ${ }^{2}$ for a discrete charge model gives a curve (not shown here) that is indistinguishable from the dashed line. A point to note is that the discrete charge distribution significantly lowers the OSF theoretical $q_{\mathrm{el}}$ for $C_{\mathrm{s}}$ above $0.2 \mathrm{M}$ where $q$ of the hyaluronate chain is dominated by $q_{0}$. In short, all the above-mentioned theories fail to describe the present $q_{\mathrm{el}}$ data and thus appear to be only applicable to (long) intrinsically stiff chains.

Schmidt and coworkers ${ }^{9,36}$ computed $q_{\mathrm{el}}$ for flexible polyelectrolytes using a mean-field approach and Koyama's distribution function ${ }^{37}$ for the end-to-end vector of a wormlike chain, and showed that $q_{\mathrm{el}}$ can be much larger than OSF's values unless the ionic strength is extremely low. Though this appears to be in line with our data, their theoretical $q_{\mathrm{el}}$ contains contributions from electrostatic excluded-volume effects.

\section{Excluded-Volume Strength}

The circles in Figure 7 show that $B$ for Na hyaluronate in aqueous $\mathrm{NaCl}$ increases almost linearly with increasing $C_{\mathrm{s}}^{-1 / 2}$. If extended to infinite ionic strength, this linear relation yields a value of about $1 \mathrm{~nm}$ for the nonionic excluded-volume strength, which is one order of magnitude smaller than the $B$ values at $C_{\mathrm{s}}{ }^{-1 / 2}>7 \mathrm{M}^{-1 / 2}$ (i.e., at $\left.C_{\mathrm{s}}<0.02 \mathrm{M}\right)$. Thus, $\beta\left(=a^{2} B\right)$ in this low $C_{\mathrm{s}}$ region is dominated by its electrostatic contribution $\beta_{\mathrm{el}}$. As is well known, the conventional bead-bead interaction model in the Debye-Hückel approximation gives $\beta_{\mathrm{el}}$ values that are a few orders of magnitude larger than experimental estimates at such low ionic strength. ${ }^{13,38,39}$ This is also the case for $\mathrm{Na}$ hyaluronate (the charge spacing is $1.0 \mathrm{~nm}$ ), for which we found that the dis-

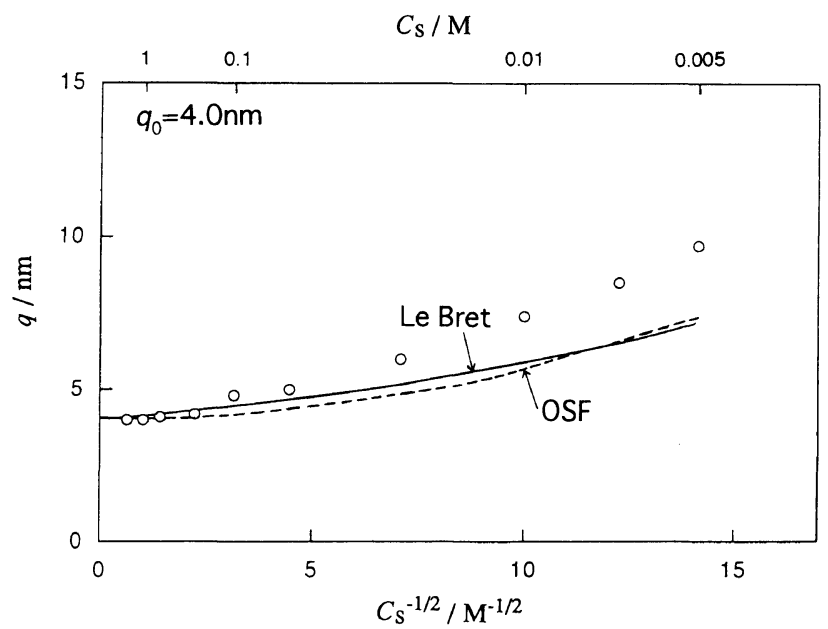

Figure 6. Experimental values (the circles) for the total persistence length of $\mathrm{Na}$ hyaluronate in aqueous $\mathrm{NaCl}$ compared with the Le Bret theory $^{3}$ (the solid line) and the Odijk-Skolnick-Fixman theory ${ }^{1,2}$ (the dashed line). The intrinsic persistence length is taken to be $4.0 \mathrm{~nm}$ irrespective of $C_{\mathrm{s}}$. 


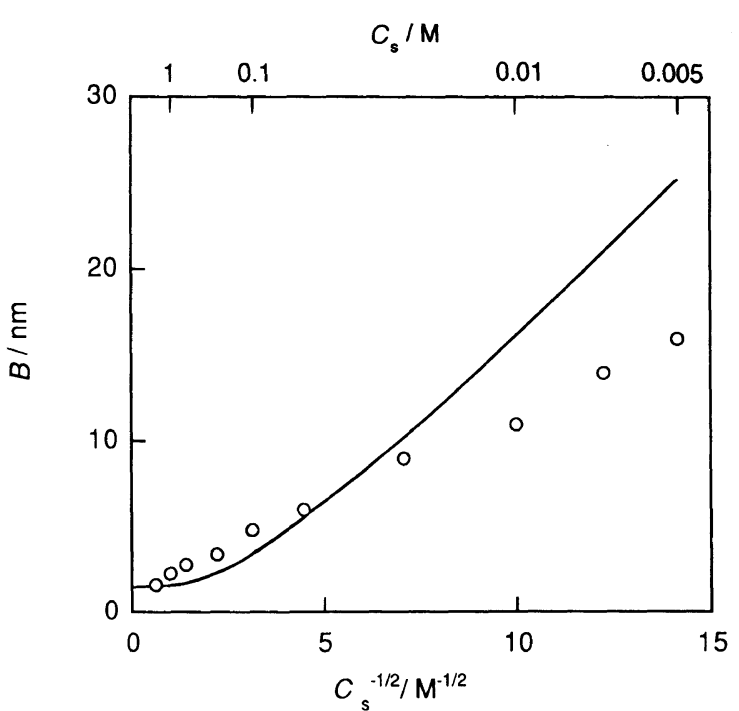

Figure 7. Values of the excluded-volume strength $B$ for $\mathrm{Na}$ hyaluronate (the circles) compared with the Fixman-Skolnick theory ${ }^{13}$ (the curve) for $\sigma=1.0 \mathrm{~nm}^{-1}$ and $d_{\mathrm{c}}=1.0 \mathrm{~nm}$.

crepancy amounts to one order of magnitude at $C_{\mathrm{s}}=$ $0.005 \mathrm{M}$. This factor of 10 is too large to be attributed to the Debye-Hückel approximation, since the charge parameter for the polysaccharide is only about 0.7 .

Fixman and Skolnick, ${ }^{13}$ evaluating the excluded volume $\beta^{\prime}$ for a pair of rodlike segments with line charge distribution in the Debye-Hückel approximation, explained the early observed large discrepancies ${ }^{38,39}$ in $\beta_{\mathrm{el}}$ as due to the direct application of the bead-bead interaction model to long-range charge interactions at low ionic strength. Since, as mentioned in the Introduction, their theory has often been used for evaluation of $z$ in the study of $q_{\mathrm{el}},{ }^{10-12}$ its comparison with our $B$ values (at $C_{\mathrm{s}} \leq 0.01 \mathrm{M}$ ) is of practical interest.

If $\beta^{\prime}$ for a pair of segments of length $l$, each consisting of $n_{0}$ beads $\left(l=n_{0} a\right)$, is equated to $n_{0}{ }^{2} \beta,{ }^{13}$ it follows that $\beta / a^{2}=\beta^{\prime} / l^{2}$. The Fixman-Skolnick theory for $\beta^{\prime}$ is then expressed in terms of $B$ as

$$
B=(\pi / 2) d_{\mathrm{c}}+(2 / \kappa) R(y)
$$

where $d_{\mathrm{c}}$ is the hard core diameter of each rodlike segment and $R(y)$ is a known function of $y$ defined by

$$
y=2 \pi \sigma^{2} Q \kappa^{-1} \exp \left(-\kappa d_{\mathrm{c}}\right)
$$

In eq 10 , the first and second terms represent the hard core and electrostatic contributions, respectively, and the attractive contribution is assumed to be negligible; note that $B$ requires no specification of $l$. If $y$ is greater than $3, R(y)$ is approximated by $R(y)=(\pi / 4)(\ln y+0.7704)$ and $B$ does not depend on $d_{\mathrm{c}}$.

The curve in Figure 7 represents eq 10 with $\sigma=$ $1.0 \mathrm{~nm}^{-1}$ and $d_{\mathrm{c}}=1.0 \mathrm{~nm}$. It comes close to the plotted points for $C_{\mathrm{s}}^{-1 / 2}$ below $8 \mathrm{M}^{-1 / 2}$. Thus we find that the Fixman-Skolnick theory allows a fairly satisfactory description of $\alpha_{\eta}{ }^{3}$ for $\mathrm{Na}$ hyaluronate at $C_{\mathrm{s}} \geq 0.05 \mathrm{M}$ if it is combined with the YSS theory. Though the theoretical $B$ values are appreciably larger than our estimates at $C_{\mathrm{s}} \leq 0.01 \mathrm{M}$, we are enable to say that the Fixman-Skolnick theory is entirely responsible for this discrepancy. Our $B$ data based on the YSS theory are inaccurate at $C_{\mathrm{s}}=0.0067$ and $0.005 \mathrm{M}$.

\section{CONCLUSIONS}

The molecular weight dependence of $[\eta]$ for $\mathrm{Na}$ hyaluronate in aqueous $\mathrm{NaCl}$ with $C_{\mathrm{s}} \geq 0.01 \mathrm{M}$ at $25^{\circ} \mathrm{C}$ is described fairly satisfactorily by the YamakawaFujii-Yoshizaki viscosity theory ${ }^{20,21}$ for unperturbed wormlike chains combined with the Yamakawa-Stockmayer-Shimada excluded-volume theory, ${ }^{22-24}$ throughout the entire molecular weight range studied $\left(3.8 \times 10^{3}-\right.$ $\left.3.5 \times 10^{5}\right)$. At lower salt concentrations of 0.0067 and $0.005 \mathrm{M}$, however, the agreement is poor in the region of $M_{w}$ above $4 \times 10^{4}$ where (electrostatic) excludedvolume effects are significant. The electrostatic contribution to the persistence length determined from the analysis of the $[\eta]$ data is roughly $70 \%$ larger (at $\left.C_{\mathrm{s}}<0.02 \mathrm{M}\right)$ than predicted by the known theories, ${ }^{1-3}$ while the estimated values for the excluded-volume strength (or the binary cluster integral) are in moderate agreement with the Fixman-Skolnick theory ${ }^{13}$ for a pair of charged rodlike segments unless $C_{\mathrm{s}}$ is lower than $0.05 \mathrm{M}$.

Acknowledgment. This work was supported in part by a Grant-in-Aid (06403027) for Scientific Research from the Ministry of Education, Science, and Culture of Japan.

\section{REFERENCES}

1. T. Odijk, J. Polym. Sci., Polym. Phys. Ed., 15, 477 (1977).

2. J. Skolnick and M. Fixman, Macromolecules, 10, 944 (1977).

3. M. Le Bret, J. Chem. Phys., 76, 6243 (1982).

4. M. Fixman, J. Chem. Phys., 76, 6346 (1982).

5. O. Kratky and G. Porod, Rec. Trav. Chim., 68, 1106 (1949).

6. For example, M. Le Bret, J. Chem. Phys., 76, 6243 (1982).

7. M. Tricot, Macromolecules, 17, 1698 (1984).

8. M. Ragnetti and R. C. Oberthür, Colloid Polym. Sci., 264, 32 (1986).

9. S. Förster, M. Schmidt, and M. Antonietti, J. Phys. Chem., 96, 4008 (1992).

10. L. Wang and H. Yu, Macromolecules, 21, 3498 (1988).

11. S. Ghosh, X. Li, C. E. Reed, and W. F. Reed, Biopolymers, 30, 1101 (1990).

12. E. Fouissac, M. Milas, M. Rinaudo, and R. Borsali, Macromolecules, 26, 5613 (1992).

13. M. Fixman and J. Skolnick, Macromolecules, 11, 863 (1978).

14. H. Yamakawa and G. Tanaka, J. Chem. Phys., 47, 3991 (1967).

15. S. K. Gupta and W. C. Forsman, Macromolecules, 5, 779 (1972).

16. T. Yoshizaki and H. Yamakawa, Macromolecules, 13, 1518 (1980).

17. T. Norisuye, Prog. Polym. Sci., 18, 543 (1993).

18. R. L. Cleland, Biopolymers, 23, 647 (1984).

19. K. Hayashi, K. Tsutsumi, F. Nakajima, T. Norisuye, and A. Teramoto, Macromolecules, 28, 3824 (1995).

20. H. Yamakawa and M. Fujii, Macromolecules, 7, 128 (1974).

21. H. Yamakawa and T. Yoshizaki, Macromolecules, 13, 633 (1980).

22. H. Yamakawa and W. H. Stockmayer, J. Chem. Phys., 57, 2843 (1972).

23. H. Yamakawa and J. Shimada, J. Chem. Phys., 83, 2607 (1985).

24. J. Shimada and H. Yamakawa, J. Chem. Phys., 85, 591 (1986).

25. See, for flexible polymers, F. Abe, Y. Einaga, T. Yoshizaki, and H. Yamakawa, Macromolecules, 26, 1884 (1993); K. Horita, F. Abe, Y. Einaga, and H. Yamakawa, Macromolecules, 26, 5067 (1993); F. Abe, K. Horita, Y. Einaga, and H. Yamakawa, Macromolecules, 27, 725 (1994); M. Kamijo, F. Abe, Y. Einaga, and H. Yamakawa, Macromolecules, 28, 1095 (1995).

26. See, for stiff chains, T. Norisuye, A. Tsuboi, and A. Teramoto, Polym. J., 28, 357 (1996). 


\section{K. HAYASHI et al.}

27. H. Yamakawa, "Molecular Conformation and Dynamics of Macromolecules in Condensed Systems," M. Nagasawa, Ed., Elsevier: Amsterdam, 1988, p 21.

28. C. Domb and A. J. Barrett, Polymer, 17, 179 (1976).

29. A. J. Barrett, Macromolecules, 17, 1566 (1984)

30. T.Sho, T. Sato, and T. Norisuye, Biophys. Chem., 25, 307 (1986).

31. F. Abe, Y. Einaga, and H. Yamakawa, Macromolecules, 26, 1891 (1993).

32. T. Yoshizaki, I. Nitta, and H. Yamakawa, Macromolecules, 21, 165 (1988).

33. S. V. Bushin, V. N. Tsvetkov, E. B. Lysenko, and V. N.
Emel'yanov, Vysokomol. Soedin. Ser. A, 23, 2494 (1981).

34. M. Bohdanecký, Macromolecules, 16, 1483 (1983).

35. G. S. Manning, J. Chem. Phys., 51, 924 (1969).

36. M. Schimidt, Macromolecules, 24, 5361 (1991).

37. R. Koyama, J. Phys. Soc. Jpn., 34, 1029 (1973).

38. I. Noda, T. Tsuge, and M. Nagasawa, J. Phys. Chem., 74, 710 (1970).

39. M. Nagasawa and A. Takahashi, "Light Scattering from Polymer Solutions," M. B. Huglin, Ed., Academic Press, New York, N. Y., 1972, Chapter 16. 